\title{
Female Pheromone and Physical Exercise Improve Endocrine Status in Elderly Japanese Men
}

\author{
Akira Tamagawa ${ }^{1)}$, Ludmila A. Gerlinskaya ${ }^{2)}$, Ryoichi Nagatomi ${ }^{1)}$, Mikhail P. Moshkin ${ }^{2)}$ \\ 1) Department Medicine and Science in Sports and Exercise, Graduate School of Medicine, Tohoku University, Sendai (Japan) \\ 2) Institute of Systematics and Ecology of Animals, Siberian Branch of RAS, Novosibirsk (Russia)
}

\begin{abstract}
BACKGROUNDS: Declines in muscle mass with age are closely related to changes in the balance between anabolic and catabolic hormones, probably due to the progressive decrease in circulating testosterone. There are some factors to affect the anabolic to catabolic hormone ratio. For example, anabolic to catabolic hormone ratio increases transitorily just after exercise. In addition, an increase in salivary testosterone in men exposed to female pheromones has been reported. Although exercise tolerability and olfactory perception decline with age, we can not exclude the possibility of the adaptation for exercise training and of the sensitivity for pheromone exposure in elderly men.

OBJECTIVE: To examine whether exposure to female pheromones and physical exercise can modulate anabolic and catabolic endocrine status in elderly Japanese men.

DESIGN: A random cohort, double-blind, placebo-controlled trial.

SETTING: Investigators tested subjects at community center in Tomiya Town, Miyagi, Japan.

PARTICIPANTS: Twelve 52 to 74 y-old Japanese men participating in a 5-week exercise class.

MEASUREMENTS: From the second to fifth exercise class periods a commercially available pheromone (Osmopherine) was applied to the upper lip of 6 men before exercise and continued as volatile molecules were released from necklace worn continuously. Another 6 men received the pheromone carrier gel on their upper lip and a necklace with only the vehicle. Saliva samples were collected before and after each exercise class to measure testosterone and cortisol concentrations. The daily physical activity level for each subject was assessed by an accelerometer.

RESULTS: Pheromone exposure and exercise classes led to a significant increase in the pre-exercise testosterone to the cortisol ratio $(F 1,39=4.8, P=0.035 F 4,39=12.0, P<0.001$, respectively). However, pheromone exposure did not affect the activity levels of participants.

CONCLUSION: Female pheromone exposure in combination with exercise classes modulated endocrine effects in older men.
\end{abstract}

$\boldsymbol{K E} \boldsymbol{Y} \boldsymbol{W O R D S}$ : aging, testosterone, cortisol ratio, exercise, female pheromone

\section{Introduction}

Declines in muscle mass with age are closely related to changes in the balance between anabolic (e.g., androgens, GH, IGF-I) and catabolic (cortisol) hormones, probably due to the progressive decrease in circulating testosterone. ${ }^{1-4)}$ Sarcopenia related to age results in a decrease of muscle strength, bone density, glucose tolerance and increased fatigability. ${ }^{5-7)}$ Low levels of plasma testosterone also negatively influence mental performance and psychological status of older men. ${ }^{8,9)}$ Testosterone supplements can correct physical and mental disability, but hormonal therapy has unfavorable effects, including gonad dysfunction, elevated hematocrit, increased prostate size. ${ }^{10-12)}$

The side effects of testosterone supplements stimulated studies directed to working up drug-free approaches to improve the ratio of anabolic to catabolic hormones. One is physical exercise. ${ }^{13)}$ Comparative studies of physically active and sedentary people demonstrated that lifestyle often, but not always, correlates with endocrine status in older man. ${ }^{7,14)}$ Anyway special training courses designed to improve the physical condition of elderly people had contradictory endocrine effects. Although anabolic to catabolic hormone ratio increased transitorily just after exercise, ${ }^{15)}$ no longterm course was found effective for improving endocrine status. For example, 16 weeks of strength training lead to an increase in maximal strength and muscle power output of the arm and leg muscles in older men, but there were no changes in serum testosterone. ${ }^{16)}$

Many experimental studies on mammals have found unequivocal evidence that smelling female sex pheromones can result in activation of the male hypothalamic pituitary gonad system. ${ }^{17-19)}$ Most of these studies were performed for rodents which were delicate sense of smell, considered "macro-osmatics". The human olfactory degenerates in comparison with the rodents, considered "micro-osmatics". Although humans are referred to as "microosmatics", some authors believe that airborne chemical signals play an important role in non-verbal communication between people. ${ }^{20-22)}$ Evidence of modulations by pheromone exposure of the human endocrine system, autonomous nervous system, mood 
and other behavioral traits have been summarized in several review papers published during the last 5 years. ${ }^{23,24)}$ In particular, Grammer and Jutte ${ }^{25)}$ reported an increase in salivary testosterone in men exposed to copulins, which are described as female pheromones.

Relatively young men and women were the subjects in most the previous studies. Although olfactory perception declines with age, we can not exclude the possible somatic and psychological effects of sex pheromones on the elderly. Recently, Friebely and Rako ${ }^{26)}$ showed changes in the socio-sexual behavior in postmenopausal woman (about 57 years old), who habitually wore a fragrance "spiked" with a female pheromone manufactured by the Athena Institute for Women's Wellness Research, Chester Springs, PA (code - 25 of pheromone formula). $68.2 \%$ of the women, who received pheromone, reported increased incidences of petting, kissing, and affection. This was significantly higher than the only $40.9 \%$ increase reported in the controls who received the placebo. These data indicate that the contemporary partners of these women who wore the fragrance were susceptible to female pheromones.

Based on the studies of Grammer and Jutte ${ }^{25}$ and Friebely and Rako ${ }^{26)}$, we hypothesized an increase in the anabolic to catabolic hormone ratio in elderly men exposed to female pheromones. Because an organized male group provides suitable conditions for a controlled pheromone experiment, we studied the effects of pheromones on the hormones of elderly men who participates weekly exercise classes on Tuesdays. Salivary testosterone and cortisol were measured to calculate the anabolic to catabolic hormone ratio. The concentration of these hormones in saliva is strongly correlated with the variations of bioavailable free testosterone and free cortisol in blood plasma. ${ }^{27,28)}$

\section{Methods}

\section{Subjects}

Twelve older males taking a standard physical training course volunteered with informed consent (reviewed by the Ethics Committee of Tohoku University:No2006-282) to participate in our study. The participants ranged from 52 to 74 years old (mean \pm SD $64 \pm 5$ y). During the 5 weeks of our study, subjects had a 90 min exercise class on Tuesdays from 1900 to 2030 (local time). We used the first week to collect baseline data. From the second to the fifth class, 6 subjects selected at random were exposed to the pheromone. The other 6 were exposed to the placebo. Just before and after the classes, we collected within 15 min saliva samples to assess the pre- and post-exercise concentrations of testosterone and cortisol. Following a double-blind methodology, we did not inform the participants what type of exposure (pheromone or placebo) they received. Technical assistants who assessed the hormone concentrations were also blinded to the sample origin. In the $4^{\text {th }}$ week of training, 3 participants missed the exercise class due to their having influenza. Those participants ( 2 from the pheromone group and 1 from the placebo group) were excluded from the analysis.

\section{Pheromone}

We used a commercially available pheromone preparation produced by Vevy Europe (Genoa, Italy) sold under the name Osmopherine. According to information from the company, Osmopherine belongs to the class of female sex pheromones (copulin). We used $0.02 \%$ Osmoperine diluted in alcohol (96\%) for the necklace vials. And one part of the Osmopherine solution into 9 parts of carrier gel (Aroma and Life Co., Japan) was prepared for applications to the upper lip. Osmopherine diluted in the carrier gel was applied to the upper lip of the pheromone-exposed men, just before class from week 2 through 5 of the exercise classes. The upper lip of men in the control group was greased by pure carrier gel, according to the same. After the classes, men of both groups were each given a necklace capsule with pure alcohol (control group) or Osmopherine diluted in alcohol. They wore the capsules at all times until the end of the experiment. We also provided each man with a vial containing alcohol or diluted pheromone. The participants were instructed to add 5 to 6 drops of fluid from the vial to the necklace capsule each morning.

\section{Activity}

The exercise classes, under the guidance of an experienced instructor, consisted of $20 \mathrm{~min}$ of stretching, a 10-min brisk walk, 10 min of strength training, $40 \mathrm{~min}$ of badminton and a $10-\mathrm{min}$ cool down. Throughout the experiment, each subject wore an accelerometer (Knez Lifecorder EX activity monitor; Suzuken Co., Nagoya Japan) which measured the intensity and time spent on physical activity throughout daily life. We evaluated the activity of daily life, energy expenditure and step counts.

\section{Salivary testosterone and cortisol}

Subjects were asked to spit about $1.5 \mathrm{ml}$ of saliva into a plastic tube after oral rinsing with distilled water. Following the recommendations of Granger, et al. ${ }^{29)}$, we visually inspected the saliva samples for discoloration by blood. Three samples showing blood were rejected. Fortunately, these were samples from subjects who were excluded from analysis (see Subjects). The samples were centrifuged at $3000 \mathrm{rpm}$ for $15 \mathrm{~min}$. About $1 \mathrm{ml}$ of each sample was placed into an Eppendorf tube and stored at $-20^{\circ} \mathrm{C}$. The samples were tested using an immuno-enzyme method with commercial kits (SteroidIFA-cortisol-01 and SteroidIFAtestosterone-1, ALKOR Bio, Saint-Petersburg, Russia) with enough sensitivity to detect testosterone and cortisol in $100 \mu \mathrm{l}$ saliva samples without previous any necessary extraction step. The measurements were made according to the kit instructions. Saliva free of steroids was used as a vehicle for the standard solutions of pure cortisol (Sigma, USA) and pure testosterone (WAKO, Japan). The anabolic to catabolic ratio was calculated as: (testosterone/cortisol) $* 1000 .{ }^{30)}$

\section{Statistical Analysis}

We used a two-way ANOVA to evaluate the effects of experimental group and weeks into training on activity and hormone concentrations. We used the Student's $t$-test (for normally distributed data) and Mann-Whitney's $U$ test (for skewed data) to compare the Osmopherine and placebo groups. Data are reported as mean $\pm \mathrm{SE}$. 


\section{Results}

\section{Activity}

On days with exercise classes, the number of steps and energy expenditure of the men's activity significantly exceeded the of steps taken and the energy expenditure registered on other (rest) days (Figure 1). In the two-way ANOVA, we found that average daily activity (number of steps) and energy cost of activity $(\mathrm{kcal} / \mathrm{kg}$ ) registered during rest days (6 days between exercise days) did not depend on either the number of days into the training $(F 4,39=0.45, P=0.77 ; F 4,39=0.37, P=0.83)$ or their pheromone exposure $(F 1,39=0.96, P=0.33 ; F 1,39=0.50, P=0.48$, correspondently). Further, neither factor (number of weeks into training or pheromone exposure) affected the number of steps or energy cost of activity $(F 3,31=0.79, P=0.51$ and $F 1,31=0.82, P=0.37$ for number of steps, and $F 3,31=0.47, P=0.70$ and $F 1,31=1.18, P=0.29$ for energy expenditure) on days with exercise classes.
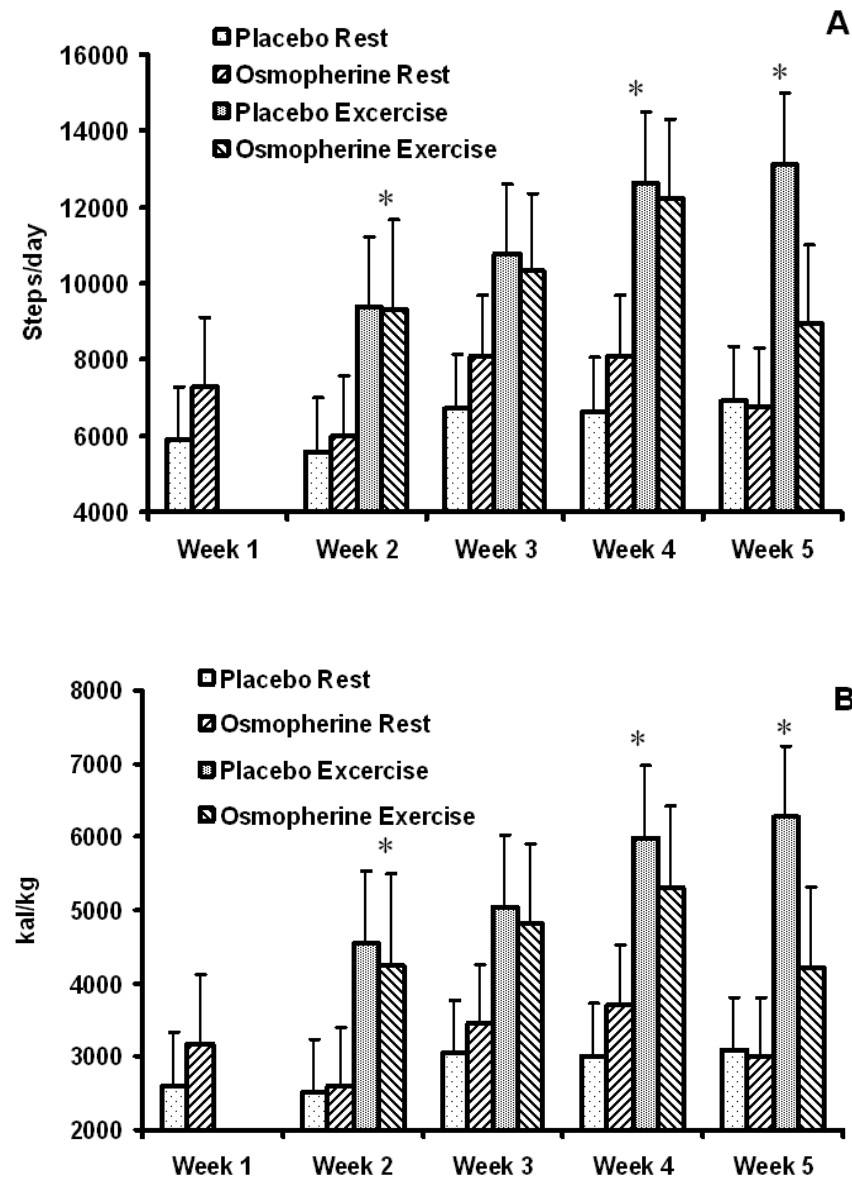

Fig. 1. Diurnal activity (A) and energy expenditure from activity (B) during an exercise training course in older men exposed to placebo or Osmopherine.

Placebo Rest and Osmopherine Rest - average daily activity or energy expenditure during 6 days between exercise classes.

Placebo Exercise and Osmopherine Exercise - daily activity or energy expenditure on the days of exercise classes, including activity and energy expenditure recorded during class.

* $\mathrm{P} \leq 0.05$ between day of the exercise class and rest days in the corresponding week (Student's paired $t$-test).

\section{Testosterone}

Using a two-way ANOVA, we calculated the influence of the date of sampling $(F 4,39=3.05, P=0.029)$ and pheromone exposure $(F 1,39=1.86, P=0.18)$ on the pre-exercise concentration of testosterone. Although there was no statistically significant effect of the Osmopherine application, the only increase in salivary testosterone during the training occurred in the group exposed to pheromone (Figure 2). Those subjects showed higher concentrations of testosterone compared with the baseline level (February 15), beginning from one week after Osmopherine application until the end of the study (March 15).

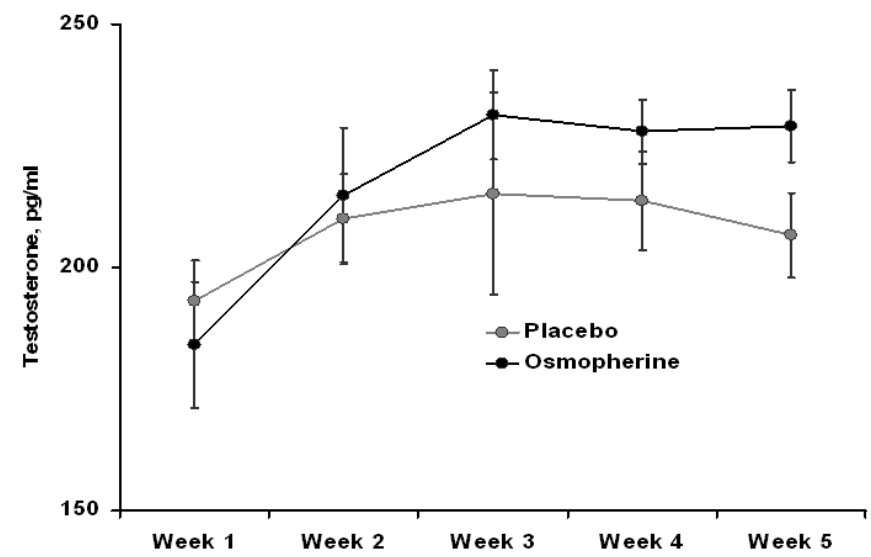

Fig. 2. Pre-exercise salivary testosterone in older men exposed to placebo or Osmopherine.

The concentration of testosterone in the saliva sampled after exercise did not differ from the pre-exercise levels $(212 \pm 4 \mathrm{pg} / \mathrm{ml}$ and $208 \pm 5 \mathrm{pg} / \mathrm{ml}$, respectively, $t=0.85, \mathrm{df}=88, P=0.39$ ). The differences in the concentrations of testosterone in the post-exercise samples wee caused by the number of weeks into training $(F 4,39=5.84$, $P=0.001)$ and did not depend on pheromone exposure $(F 1,39=$ $1.07, P=0.20$ ). In groups exposed to either Osmopherine or the placebo, the post-exercise testosterone levels exceeded the initial levels following the $2^{\text {nd }}, 3^{\text {rd }}$, and $4^{\text {th }}$ exercise classes (Figure 3 ).

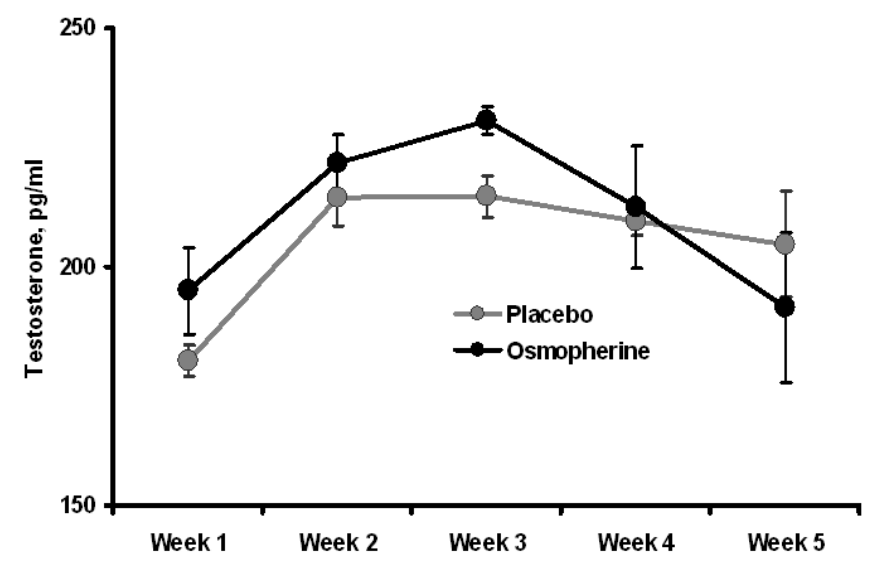

Fig. 3. Post-exercise testosterone in saliva of older men exposed to placebo or Osmopherine. 


\section{Cortisol}

We found no statistically significant difference in the concentrations of cortisol between samples collected before and after classes, perhaps because of the low intensity of exercise $(5.94 \pm 0.36$ $\mathrm{ng} / \mathrm{ml}$ and $6.18 \pm 0.41 \mathrm{ng} / \mathrm{ml}$, respectively, $t=0.44, \mathrm{df}=88, P=0.66$ ). The two-way ANOVA showed that the number of weeks into the training course had an influence on the salivary cortisol levels in samples collected before and after exercise $(F 4,35=4.39, P=0.006$ and $F 4,39=2.89, P=0036$,respectively). There was no significant effect of the pheromone on the concentration of cortisol in saliva samples collected before $(F 1,39=1.71, P=0.20)$ or after exercise $(F 1,39=0.01, P=0.93)$. In both groups, the concentrations of cortisol in saliva samples collected before and after exercise were significantly lower on the day of the last exercise class (March 15) than in the first day (February 15) of the training course (Figure 4, Figure 5).

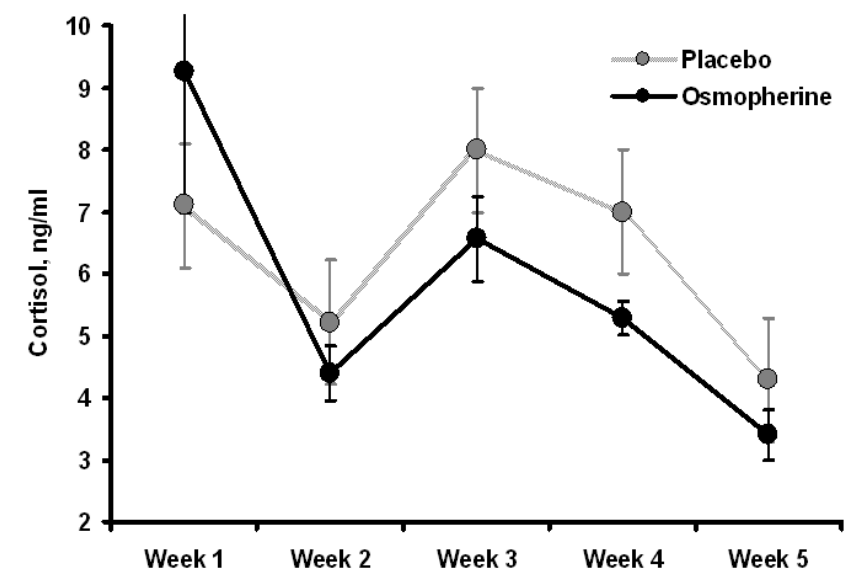

Fig. 4. Pre-exercise cortisol in saliva of older men exposed to placebo or Osmopherine.

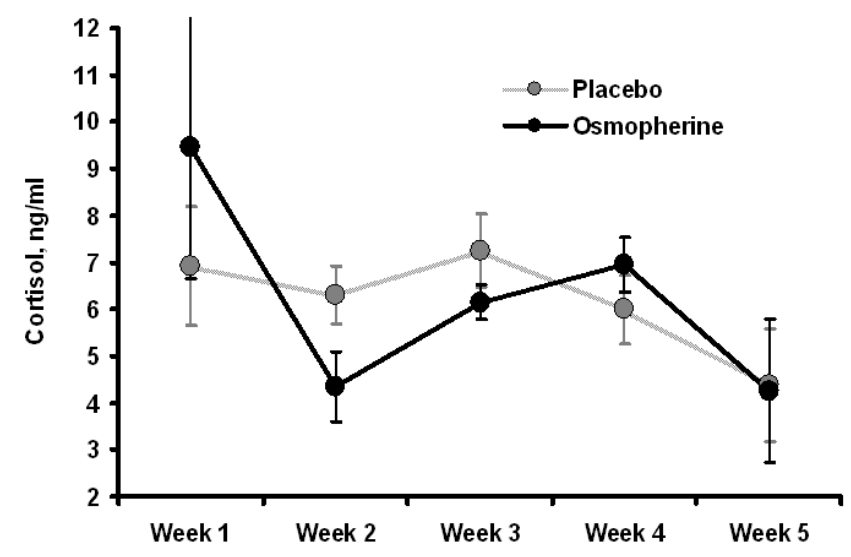

Fig. 5. Post-exercise cortisol in saliva of older men exposed to placebo or Osmopherine.

\section{Testosterone to cortisol ratio ( $T / C$ ratio)}

Both the number of weeks into the training course and pheromone exposure were statistically significant factors for the pre-exercise testosterone to cortisol ratio $(F 4,39=12.0, P<0.001$ and $F 1,39=4.8, P=0.035$, respectively). This ratio increased from the first to last exercise class (Figure 6). In samples collected before the $4^{\text {th }}$ and $5^{\text {th }}$ classes, the testosterone to cortisol ratio was significantly higher in the Osmopherine than in the Placebo groups. Two-ways ANOVA revealed a statistical significant effect of the number of weeks into the training course on the testosterone to cortisol ratio, assessed just after exercise classes $(F 4,39=5.2$, $P=0.002$ ). But there was no a statistical significance effect of the pheromone exposure on the testosterone to cortisol ratio, assessed just after exercise classes $(F 1,39=0.7, P=0.41)$. As in post-exercise samples, this ratio increased from the first to the last exercise class in the group exposed to the placebo (Figure 7). In men exposed to Osmopherine, the testosterone to cortisol ratio in general rose after the $2^{\text {nd }}$ class, then declined to its initial level and increased again after the last class.

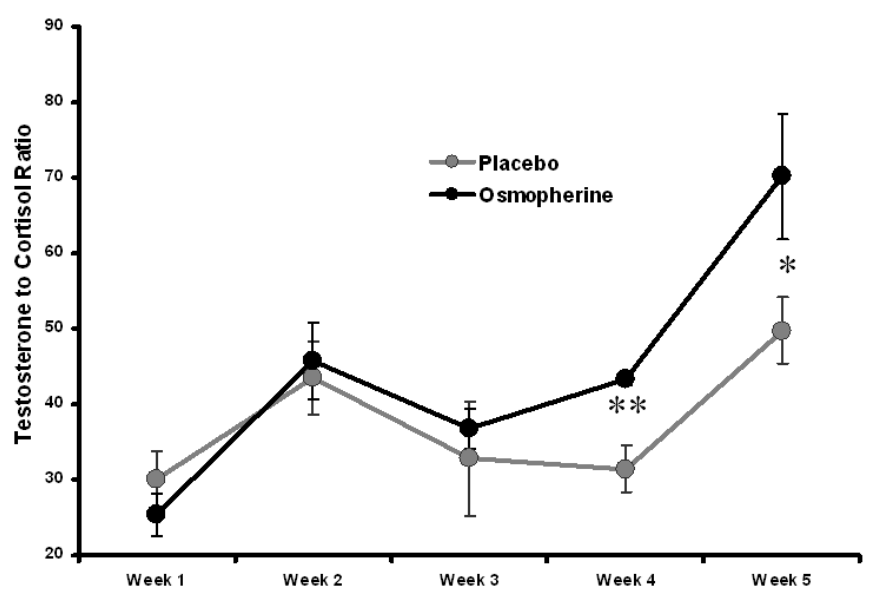

Fig. 6. Pre-exercise testosterone to cortisol ratio of older men exposed to placebo or Osmopherine.

* $\mathrm{P}=0.05$, ** $\mathrm{P}=0.01$ between placebo and Osmopherine groups (Student's $t$-test).

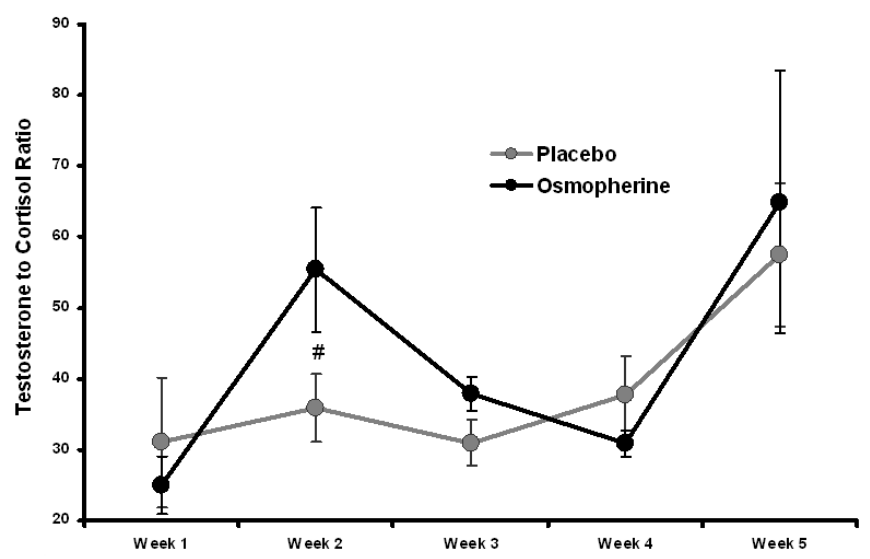

Fig. 7. Post-exercise testosterone to cortisol ratio of older men exposed to placebo or Osmopherine.

\# P $=0.07$ between placebo and Osmopherine groups (Student's $t$ test). 


\section{Discussion}

In this study, we examined the effects of female pheromone exposure and physical exercise on the anabolic and catabolic endocrine status in elderly Japanese men. This training program consisted of moderate exercise once a week. This level of exercise did lead to a statistically significantly increase in the anabolic to catabolic balance in the older men due to opposite changes in levels of salivary testosterone and cortisol, especially in samples collected before exercise. The endocrine effect of regular exercise was more prominent when participants were exposed to a female pheromone along with training. The increased anabolic to catabolic ratio in men exposed to Osmopherine also resulted in a significant increase in testosterone and a decrease in cortisol.

The other hand, we were not able to observe the same changes from the post-exercise concentrations of testosterone and cortisol. The post-exercise testosterone to cortisol ratio declined at week 3 and week 4 in comparisons with week 2 in men exposed to Osmopherine. The cause of decline at week 3 and week 4 was the significant decrease of the cortisol in week 2. The low level of cortisol at week 2 provided the elevation of testosterone to cortisol ratio at week 2 . It could be the effect of unpredictable response to the first application of Osmopherine or to other non-controlable factors.

The influence of female pheromones on the secretions from male gonads has been reported in several papers on animals, but in only a few studies on humans. Similar to our study, application of copulins (a female pheromone) was found to increase salivary testosterone in men. ${ }^{25)}$ Another human pheromone, vomeropherin pregna-4,20-diene-3,6-dione (PDD), decreases serum LH and testosterone when delivered locally to the male human nasal cavity. ${ }^{31)}$ The opposite endocrine reactions of copulins and PDD could be a reflection of the different origins of these chemicals (sweat from the armpit and vaginal secretion).

Causal explanations for the endocrine effect of chemical signals are based on the perception of pheromones by the vomero-nasal organ (VNO), which projects into the hypothalamus. ${ }^{32.33)}$ Although the existence and olfactory function of the VNO in adults are still debated, ${ }^{24)}$ several recent papers have reported VNO-related responses to sex pheromones in humans. For example, the electrophysiological response of VNO to nebulized sex pheromones was demonstrated in studies on humans, ${ }^{31,34)}$ and positron emission tomography was used to show that pheromones activate the hypothalamic area in healthy people. ${ }^{35)}$ This effect occurs if female pheromones are presented to men, and male pheromones to women.

However, the subjects in those reports were significantly younger than the men in our experiment. Our finding that older men retain the capability to generate an endocrine response to female pheromones confirms the behavioral observations of Friebely and Rako. ${ }^{26)}$ They reported that the addition of female pheromones to common perfume increased the sexual attraction of postmenopausal women. This effect was assessed by documenting behavioral changes in their contemporary partners.

In our study, we observed significant increase of testosterone by female pheromone exposure in the combination with exercise class in older men. Our data suggests the possibility of pheromone exposure to improve their anabolic to catabolic ratio. However, we did not evaluate their muscle mass and the sole effect of pheromone exposure. We need further researches to demonstrate the obious effect of pheromone exposure on muscle atrophy.

\section{Acknowledgements}

We thank Irene Kolossova and Kate Litvinova for their valuable assistance in measuring salivary steroids. Russian Foundation for Basic Research (grant 02-04-49253) and the Heiwa Nakajima Foundation supported this work. We very appreciate careful job of Jon Moon and John Burns, which corrections dramatically improve final version of the manuscript. 


\section{References}

1) Copeland KC, Colletti RB, Devlin JT, et al. The relationship between insulin-like growth factor-I and aging. Metabolism 1990;39:584-587.

2) Longcope C, Goldfield SRW, Brambilla DJ, et al. Androgens, estrogens, and sex hormone-binding globulin in middle-aged men. J Clin Endocrinol Metab 1990;71:1442-1446.

3) Hakkinen K, Pakarinen A. Muscle strength and serum testosterone, cortisol, and SHBG concentration in middle-aged and elderly men and women. Acta Physiol Scand 1993;148:199-207.

4) Rosen CJ, Conover C. Growth hormone/insulin-like growth factor-I axis in aging: a summary of a National Institute on Aging-sponsored symposium. J Clin Endocrinol Metab 1997;82:3919-3922.

5) Kamel HK. Sarcopenia and aging. Nutr Rev 2003;61:157-167.

6) Evans WJ Protein nutrition, exercise and aging. J Am Coll Nutr 2004;23:601S-609S.

7) Szulc P, Duboeuf F, Marchand F, et al. Hormonal and lifestyle determinants of appendicular skeletal muscle mass in men: the MINOS study. Am J Clin Nutr 2004;80:496-503.

8) Matsumoto AM. Andropause. Clinical implications of the decline in serum testosterone levels with aging in men. J Gerontol Med Sci 2002;57A:M76-M99.

9) Moffat SD, Zonderman AB, Metter EJ, et al. Longitudinal assessment of serum free testosterone concentration predicts memory performance and cognitive status in elderly men. J Clin Endocrinol Metab 2002;87:50015007.

10) Gruenewald DA, Matsumoto AM. Testosterone supplementation therapy for older men: potential benefits and risks. J Am Geriatr Soc 2003;51:101-105.

11) Schroeder ET, Zheng L, Ong MD, et al. Effects of androgen therapy on adipose tissue and metabolism in older men. J Clin Endocrinol Metab 2004;89:4863-4872.

12) Cherrier MM, Plymate S, Mohan S, et al. Relationship between testosterone supplementation and insulin-like growth factor-I levels and cognition in healthy older men. Psychoneuroendocrinology 2004;29:65-82.

13) Greenlund LJ, Nair KS Sarcopenia - consequences, mechanisms, and potential therapies. Mech Age Dev 2003;124:287-299.

14) Tissandier O, Peres G, Fiet J, et al. Testosterone, dehydroepiandrosterone, insulin-like growth factor 1 , and insulin in sedentary and physically trained aged men. Eur J Appl Physiol $2001 ; 85: 177-184$.

15) Kostka T, Patricot MC, Mathian B, et al. Anabolic and catabolic hormonal responses to experimental two-set low-volume resistance exercise in sedentary and active elderly people. Aging Clin Exp Res 2003:15:123-130

16) Izquierdo M, Hakkinen $K$, Ibanez J, et al. Effects of strength training on muscle power and serum hormones in middle-aged and older men. J Appl Physiol 2001;90:1497-1507.

17) Bronson FH. The reproductive ecology of the house mouse. Quarterly Rev Biol 1979;54:265-299.
18) Graham JM, Desjardins C. Classical conditioning: Induction of luteinizing hormone and testosterone secretion in anticipation of sexual activity. Science 1980;210:1039-1041.

19) Moshkin MP, Kolosova IE, Novikov EA, et al. Co-Modulation of The Immune Function and The Reproductive Chemosignals. Asian-Aust J Anim Sci 2001;4 (Special Issue):43-51.

20) Grammer K. Strangers meet: laughter and nonverbal signs of interest in opposite-sex encounters. J Nonverb Behav 1991;14:209-236.

21) Grammer K, Kruck K, Juette A, et al. Non-verbal behavior as courtship signals: The role of control and choice in selecting partners. Evol Hum Behav 2000;21:371-390

22) Kohl JV, Atzmueller M, Fink B, et al. Human pheromones: Integrating neuroendocrinology and ethology. Neuroendocr Let 2001;22:309-321.

23) Grammer K, Fink B, Renninger L. Dynamic systems and inferential information processing in human communication. Neuroendocrinology Letters 2002;23 (Suppl. 4):103-110.

24) Wysocki CJ, Preti G. Facts, fallacies, fears and frustrations with human pheromones. The Anatomical Record 2004 ; Part A. 281A:1201-1211.

25) Grammer K, Jutte A. Battle of odors: significance of pheromones for human reproduction. Gynakol Geburtshilfliche Rundsch 1997;37:150153.

26) Friebely J, Rako S. Pheromonal influences on sociosexual behavior in postmenopausal women. J Sex Res 2004;41:372-80.

27) Riad-Fahmy D, Read GF, Walker RF, et al. Steroids in saliva for assessing endocrine function. Endocrine Reviews 1982;3:367-395.

28) Sakaguci K, Hasegawa T. Analysis of salivary testosterone by liquid chromatography-tandem mass spectrometry: correlation with serum bioavailable testosterone and aging. Rinsho Byori 2005;53:388-394.

29) Granger DA, Shirtcliff EA, Booth A, et al. The "trouble" with salivary testosterone. Psychoneuroendocrinology 2004;29:1229-1240.

30) Deschenes MR, Bronson LL, Cadorette MP, et al. Aged men display blunted biorhythmic variation of muscle performance and physiological responses. J Appl Physiol 2002;92:2319-2325.

31) Monti-Bloch L, Diaz-Sanchez V, Jennings-White C, et al. Modulation of serum testosterone and autonomic function through stimulation of the male human vomeronasal organ (VNO) with pregna-4,20-diene3,6-dione. J Steroid Biochem Mol Biol 1998;65:237-242.

32) Savic I. Processing of odorous signals in humans. Brain Res Bull 2001;54(3):307-312.

33) Dulac C, Torello TA Molecular detection of pheromone signals in mammals: from genes to behavior. Nature Reviews Neuroscience 2003;4:551-562

34) Grosser BI, Monti-Bloch L, Jennings-White C, et al. Behavioral and electrophysiological effects of androstadienone, a human pheromone. Psychoneuroendocrinology 2000;25:289-299.

35) Savic I, Berlund H, Lindstrom P. Brain response to putative pheromones in homosexual men. PNAS 2005;102:7356-7361. 